\title{
A Theoretical Upper Bound for IP-Based Floorplanning
}

\author{
Guowu Yang ${ }^{1}$, Xiaoyu Song ${ }^{1}$, Hannah H. Yang ${ }^{2}$, and Fei Xie ${ }^{3}$ \\ 1 Dept. of ECE, Portland State University, Oregon, USA \\ \{guowu, song\}@ece.pdx.edu \\ 2 CAD Strategic Research Lab, Intel Corporation, Oregon, USA \\ hyang@ichips.intel.com \\ 3 Dept. Computer Science, Portland State University, Oregon, USA \\ xie@Cs.pdx.edu
}

\begin{abstract}
Floorplan is a crucial estimation task in the modern layout design of systems on chips. The paper presents a novel theoretical upper bound for slicing floorplans with soft modules. We show that, given a set of soft modules of total area $A_{\text {total }}$, maximum area $A_{\max }$, and shape flexibility $r \geq 2.25$, there exists a slicing floorplan $F$ of these modules such that $\operatorname{Area}(F) \leq \min \{1.131,(1+\beta)\} A_{\text {total }}$, where $\beta=\sqrt{\frac{A_{\max }}{2 r A_{\text {total }}}}$. Our results ameliorate the existing best results.
\end{abstract}

\section{Introduction}

With the new design paradigm of system-on-chip, systems are assembled integrating blocks of intellectual property (IP), including cores, embedded software, and bus standards as elements of systems on silicon. Due to multiple sources of components, floorplanning is an important estimation task in the modern layout design in the development of giga-transistor chips.

A floorplan dissects a rectangle into a set of non-intersecting modules, called rooms, and assigns the macro blocks into the rooms (In this paper, we only study rectangular modules, so modules are considered as rectangles). The topological relationship among the blocks is specified by the dissection. One crucial factor of most floorplanners is the representation structure which affects the effectiveness and efficiency of the optimization process directly. There are two categories of floorplans: slicing and non-slicing structures [7]. If a floorplan can be obtained by recursively cutting a rectangle into two parts by either a vertical or a horizontal line, the floorplan has a slicing structure. Otherwise, the floorplan has a non-slicing structure.

Slicing structure provides a simple way for optimizing the block orientations, defining reasonable channels in global routing and appropriately ordering the channels during detailed routing. Due to its nice properties, such as slicing tree [3], Polish expression [2], polynomial algorithms for special cases $[4,5]$, etc., it is easier to design efficient strategies to search for optimal slicing floorplans [2]. And some fast algorithms were given $[10,11]$. Even if the general floorplan is not sliceable, at the initial design stage, the floorplan can be adjusted to have a slicing one by increasing space to have a fast estimation [6]. The only possible disadvantage of slicing floorplans is that even the optimal one may not pack the modules tightly [1]. Although, there are empirical evidences showing that slicing floorplans are quite good in packing modules tightly, it is important to have assurance of their performance by mathematical analysis [1]. 
Floorplan is a challenging problem in VLSI layout design. Pan [13] proved the nonslicing floorplan is NP-complete. In the early stage of the VLSI physical design, most of the modules are not designed and so are flexible in shape, i.e., soft modules. Therefore the early reliable estimation for the areas with soft modules is helpful for the design $[1,8,9]$. In recent years, IP soft modules have been widely used in ASIC design industry [14]. In the early planning stage, not all block information is available. Some blocks are not yet designed, thus being flexible in shape. The dimension of a soft block is based on several previous implementations of the block in a library. As such, the internal logic and layout structure of a building block may have a set of alternative choices. The shape of a soft module can be changed as long as the area remains a constant and the aspect ratio is within a given range.

Recently, some excellent theoretical study [1,8] has been performed. Young and Wong [1] proved that, given a set of modules with flexibility in shape, there exists a slicing floorplan $F$ such that $\operatorname{area}(F) \leq \min \left\{\left(1+\frac{1}{\lfloor\sqrt{r}\rfloor}\right), \frac{5}{4},(1+\alpha)\right\} A_{\text {total }}$, where $\alpha=\sqrt{\frac{2 A_{\max }}{r A_{\text {total }}}}, A_{\text {total }}$ is the total area of all the modules, $A_{\max }$ is the maximum module area, and $r \geq 2$ is the shape flexibility of each module. Note that if we do not consider the ratio of the maximum module area to the total area, the result is area $(F) \leq(1+$ $\left.\frac{1}{[\sqrt{r}]}\right) A_{\text {total }}$ with $r \geq 16$, or area $(F) \leq 1.25 A_{\text {total }}$ with $2 \leq r \leq 16$. Otherwise, the result is area $(F) \leq(1+\alpha) A_{\text {total }}$. Peixoto et al. [8] gave a more delicate upper bound based the same packing strategy but detailed analysis, area $(F) \leq\left(1+\frac{1}{\lfloor\sqrt{r}\rfloor^{\gamma-1}}\right) A_{\text {total }}$ where $\gamma$ is the smallest $j \geq 2$ such that group $j$ (The grouping in [8] is different from ours) is not empty. When $\gamma=1$, the upper bound in [8] is the same as in [1], and this upper bound is usable only when $r \geq 4$.

In this paper, we show that, given a set of soft rectangles of total area $A_{\text {total }}$, maximum area $A_{\max }$ and shape flexibility $r \geq 2.25$, there exists a slicing floorplan $F$ of these rectangles such that $\operatorname{Area}(F) \leq 1.131 A_{\text {total }}$. The worst relative dead space of the results of [1] is $25 \%$ for $2 \leq r \leq 16$. Our result reduces their result to about $13 \%$ for $r \geq 2.25$. Moreover, our second result is stronger than the first and the third inequalities in [1] with a slightly stronger condition of $r \geq 2.1213$. Although the parameter $r$ is slightly bigger than that in [1], it does not affect the significance of the results in practice.

\section{Main Results and Related Work}

We refer to the basic definitions in [1]. Let $R$ be a rectangle. Let height $(R)$, width $(R)$ and $\operatorname{area}(R)$ be the height, the width and the area of $R$, respectively. The ratio height $(R) /$ width $(R)$ is called as aspect ratio of $R$. A soft rectangle can have different shapes as long as the area remains the same. The shape flexibility of a soft rectangle specifies the range of its aspect ratio. A soft rectangle of area $A$ is said to have a shape flexibility $r$ if and only if $R$ can be represented by any rectangle of area provided that:

$$
\frac{1}{r} \leq \frac{\text { height }(R)}{\text { width }(R)} \leq r
$$

Given $n$ soft rectangles of area $A_{i}(i=1,2, \ldots, n)$ and a shape flexibility $r$, the problem is to obtain an upper bound on the area of the optimal slicing floorplan. Given a set 
of soft rectangles $\Pi=\left\{R_{1}, \ldots, R_{n}\right\}$, let $A_{i}$ be the area of $R_{i}, i=1, \ldots, n$. The total area $A_{\text {total }}=\sum A_{i}, A_{\text {total }}=1$, and the maximum area $A_{\max }=\max \left\{A_{1}, \ldots, A_{n}\right\}$.

Theorem 1.1 [1]. Given a set of soft rectangles of total area $A_{\text {total}}$, maximum area $A_{\max }$ and shape flexibility $r \geq 2$,

(i) there exists a slicing floorplan $\mathrm{F}$ of the rectangles such that

$$
\operatorname{area}(F) \leq \min \left\{\left(1+\frac{1}{\lfloor\sqrt{r}\rfloor}\right), \frac{5}{4},(1+\alpha)\right\} A_{\text {total }} \quad \text { where } \quad \alpha=\sqrt{\frac{2 A_{\text {max }}}{r A_{\text {total }}}}
$$

(ii) there exists the following relation:

$$
1 \leq \frac{\text { height }(F)}{\text { width }(F)} \leq \begin{cases}\left(1+\frac{1}{\lfloor\sqrt{r}\rfloor}\right) & \text { area }(F) \leq\left(1+\frac{1}{\lfloor\sqrt{r}\rfloor}\right) A_{\text {total }} \\ \frac{5}{4} & \text { area }(F) \leq \frac{5}{4} A_{\text {total }} \\ \frac{1+\alpha}{\left(1-\frac{r \alpha}{2}\right)^{2}} & \text { area }(F) \leq(1+\alpha) A_{\text {total }}\end{cases}
$$

Theorem 1.2 [8]. Given a set of soft rectangles of total area $A_{\text {total }}$, and shape flexibility $r \geq 2$, there exists a slicing floorplan $\mathrm{F}$ of the rectangles such that $\operatorname{area}(F) \leq$ $\left(1+\frac{1}{|\sqrt{r}|^{\gamma-1}}\right) A_{\text {total }}$ where $\gamma$ is the smallest $j \geq 2$ such that group $j$ (Where group $j=$ $A_{i} \mid \frac{A_{\text {total }}}{r^{j}} \leq A_{i}<\frac{A_{\text {total }}}{r^{j-1}}$ ) is not empty. (The grouping in [1,8] is different from ours).

We establish the following results.

Theorem 2. Given shape flexibility $r \geq 2.25$, there exists a slicing floorplan $F$ of $\Pi$ such that $\operatorname{Area}(F) \leq 1.131 A_{\text {total }}$, and width $(F)=\sqrt{A_{\text {total }}}, \sqrt{A_{\text {total }}} \leq \operatorname{height}(F) \leq$ $1.131 \sqrt{A_{\text {total }}}$. Namely, the relative dead space is $13.1 \%$.

Note that if we set width $(F)=\sqrt{A_{\text {total }}}$ and $r=2.25$, the optimal upper bound of the relative dead space of a floorplan is no less than $1 / 9=11.1 \%$. For example, if there are only two modules $A_{1}=8 / 9, A_{2}=1 / 9$, the optimal relative dead space is $1 / 9=11.1 \%$.

Theorem 3. Given shape flexibility $r \geq 3 \sqrt{2} / 2=2.1213$

(i) there exists a slicing floorplan $F$ of $\Pi$ such that $\operatorname{area}(F) \leq(1+\beta) A_{\text {total }}$ where $\beta=\sqrt{\frac{A_{\max }}{2 r A_{\text {total }}}}$.

(ii) $1 \leq \frac{\text { height }(F)}{\operatorname{width}(F)} \leq(1+\beta) \frac{A_{\text {total }}}{X^{2}}, X=\left[\frac{\sqrt{A_{\text {total }}}}{W}\right] \times W\left(\leq \sqrt{A_{\text {total }}}\right), W=\sqrt{\frac{r A_{\max }}{2}}$, where $X$ is the width of the floorplan $F$.

Our result improves the results in [1] with a slightly stronger condition. Theorem 2 shows the relative dead space is $13.1 \%$ with $r \geq 2.25$, while the relative dead space in [1] is $25 \%$ with $2 \leq r \leq 16$. Theorem 3 shows the relative dead space $\beta$ is as half as that in the third inequality in [1], namely, $\beta=0.5 \alpha$, and $\beta \leq \sqrt{\frac{A_{\max }}{2 A_{\text {total }}}} \cdot \frac{1}{\lfloor\sqrt{r}\rfloor}$, with $r \geq 2.1213$. Note that $\alpha$ and $\frac{1}{\lfloor\sqrt{r}\rfloor}$ are the relative dead space in [1] with $r \geq 2$ and $r \geq 16$, respectively. 
Remark 1: When the flexibility $r$ is small $(2.25 \leq r<4)$, the upper bound can be chosen as the minimum of the results in Theorem 2,3. When $r$ is big $(r<4)$, the upper bound can be chosen as the minimum of the results in Theorem 1.2,2, and 3 .

We construct a slicing floorplan $F$ of rectangles such that every rectangle satisfies the aspect ratio constraint (1) and the area of $F$ is as small as possible. We will prove the main result in Section 3. Section 4 gives the conclusion. It is obvious that the amount of dead space will decrease with the flexibility and it becomes infinitely small when the rectangles have very large flexibility. We attempt to make full use of flexibility by shaping modules. Theorem 2 will be proven in Sections 3.1, 3.2, and 3.3. We prove theorem 3 in Section 3.4.

\section{A Tighter Upper Bound}

\subsection{Classifying Modules into Groups}

Given a set $\Pi$ of soft rectangles, we classify rectangles into groups: $G_{1}, G_{2}, \ldots \ldots$, $G_{n}$.W.1.o.g, we assume that $A_{\text {total }}=1$. An area $A$ is in $G_{i}$ if and only if $\frac{1}{2^{i}} \leq A<\frac{1}{2^{i-1}}$ for $i=1,2,3, \ldots, n$. The widths of the rectangles are halved every two groups. For any module $\forall X \in \mathrm{G}_{2 \mathrm{i}}$ and $\forall Y \in \mathrm{G}_{2 \mathrm{i}+1}$, we have width $(X)=\operatorname{width}(Y)$, and for $\forall X \in G_{2 \mathrm{i}-1}$ and $\forall Y \in G_{2 \mathrm{i}}$, we have width $(X) / 2=\operatorname{width}(Y)$.

We construct a slicing floorplan $F$ as follows. Table 1 tabulates the areas, the heights and the widths of different groups. We pack the groups in the following order: group 1, groups 2 and 3, groups 4 and 5, group 6, and group $n(n \geq 7)$. At each packing step, we place the rectangles from the largest to the smallest, and on the lowest possible level. When packing groups 2 and 3, groups 4 and 5, respectively, we always compress and align a pair of modules of the same group with the same height and the same total width (Lemma 1 and Figure 1), and then place the pairs. If the number of rectangles in group $i$ $(i=2,3,4,5)$ is odd, then place the single rectangle. If the number of rectangles is even in both groups 2 and 3, they can be compressed evenly in terms of lemma 1 and can be considered as group 1 . Thus, we only need to consider the case where there is at most one rectangle in both groups 2 and 3, respectively. In the process of packing groups $2 i$ and $2 i+1,(i=1,2)$, if the tops of the modules do not surpass the unit height over 0.125 , we do not need a special shaping (just using Lemma 1). In Section 3.2, we deal with the cases that the tops of the modules surpass the unit height over 0.125 . If the tops of a pair of modules (compressed evenly in terms of lemma 1) surpass the unit height over 0.125 , we decompress them first and then shape them respectively. Group 6 may need shaping discussed in Section 3.3. Group $n(n \geq 7)$ does not need any shaping. From the packing procedure, it is not difficult to see that the final packing gives a slicing floorplan.

There are three important aspects in our packing strategy that are different from [1]. First, we classify the module areas in a finer way: partition every group after the second group in [1] into two parts with the same width. Second, we can always compress a pair of modules of the same group aligned with the same height and the same total width (Lemma 1 and Figure 1), thus compressing modules tighter. Third, in cases 2, 3 and 4 in Section 3.2, we set the width of the left empty part as a new unit to deal with the remaining rectangles.

Let $H$ be the height of a module, $\bar{H}$ the height of a shaped module and $\bar{w}$ the width of a shaped module. 
Table 1. Classification of areas

\begin{tabular}{cccc}
\hline Group & Area $A$ & Width $w$ & Height $H$ \\
\hline 1 & $1 / 2 \leq A \leq 1$ & $w=1$ & $1 / 2 \leq H \leq 1$ \\
\hline 2 & $1 / 4 \leq A<1 / 2$ & $w=1 / 2$ & $1 / 2 \leq H<1$ \\
\hline 3 & $1 / 8 \leq A<1 / 4$ & $w=1 / 2$ & $1 / 4 \leq H<1 / 2$ \\
\hline 4 & $1 / 16 \leq A<1 / 8$ & $w=1 / 4$ & $1 / 4 \leq H<1 / 2$ \\
\hline 5 & $1 / 32 \leq A<1 / 16$ & $w=1 / 4$ & $1 / 8 \leq H<1 / 4$ \\
\hline 6 & $1 / 64 \leq A<1 / 32$ & $w=1 / 8$ & $1 / 8 \leq H<1 / 4$ \\
\hline 7 & $1 / 128 \leq A<1 / 64$ & $w=1 / 8$ & $1 / 16 \leq H<1 / 8$ \\
\hline$\ldots$ & $\ldots \ldots$ & $\ldots \ldots$ & $\ldots \ldots$ \\
\hline
\end{tabular}

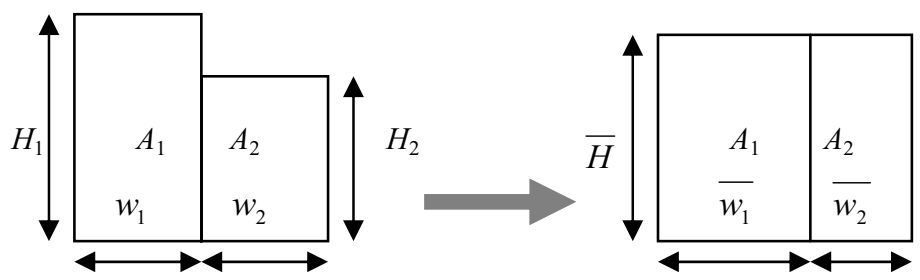

Fig. 1. Shaping in Lemma 1

Lemma 1. Any two rectangles of the same group can be aligned with the same height and the same total width.

Proof: Let $H_{1}\left(w_{1}\right)$ and $H_{2}\left(w_{2}\right)$ be the heights (widths) of the two modules, respectively (see Fig. 1). Initially, $w_{1}=w_{2}=w$.

(1) $\frac{1}{2} \leq \frac{H_{1}}{w}<1, \frac{1}{2} \leq \frac{H_{2}}{w}<1$. Consider the extreme case: $H_{1}=w, H_{2}=\frac{1}{2} w$. Set $\bar{H}=$ $\frac{1}{2}\left(H_{1}+H_{2}\right)=\frac{3}{4} w$, so we have $\overline{w_{1}}=\frac{4}{3} w, \overline{w_{2}}=\frac{2}{3} w$. We have $\overline{H w_{1}}=w^{2}=H_{1} w, \overline{H w_{2}}=$ $\frac{1}{2} w^{2}=H_{2} w, \overline{w_{1}}+\overline{w_{2}}=2 w$. This means that the area of the modules remains the same, and the total width keeps the same. And we obtain $\frac{\bar{H}}{\overline{w_{1}}}=\frac{9}{16} \in\left[\frac{4}{9}, \frac{9}{4}\right], \frac{\bar{H}}{\overline{w_{2}}}=\frac{9}{8} \in\left[\frac{4}{9}, \frac{9}{4}\right]$. Thus, the lemma holds.

(2) $1 \leq \frac{H_{1}}{w}<2,1 \leq \frac{H_{2}}{w}<2$. The proof is similar to (1).

\subsection{Packing Groups 2 to 5}

We only need to consider the case where there is at most one rectangle in both groups 2 and 3, respectively. The reason is discussed in 3.1. Groups $n(n \geq 6)$ are discussed in Section 3.3. In Section 3.2, we consider the following cases.

Case 1: No rectangle in either group 2 and or group 3.

Case 1.1: Even number of rectangles in both groups 4 and 5.

Case 1.2: Even number of rectangles in group 4 and odd number of rectangles in group 5.

Case 1.3: Odd number of rectangles in group 4 and even number of rectangles in group 5.

Case 1.4: Odd number of rectangle(s) in both groups 4 and 5.

Case 2: One rectangle in group 2 and no rectangle in group 3.

Case 3: No rectangle in group 2 and one rectangle $A$ in group 3.

Case 4: One rectangle $A_{1}$ in group 2 and one rectangle $A_{2}$ in group 3. 
Due to the space limitation, we only show case 1.1 . The detailed algorithm can be found in [15].

\section{Case 1: No Rectangle in Either Group 2 and or Group 3}

For the rectangles in group 4 with area from $1 / 9$ to $1 / 8$, set $w=1 / 2,2 / 9 \leq h<1 / 4$, and pack them first. Assume the height in group 4 is from 1/4 to 4/9. For the remaining rectangles, if the number is over one, each pair of them can be compressed evenly in terms of lemma 1 and packed together.

\section{Case 1.1: Even Number of Rectangles in Both Groups 4 and 5}

If there are a pair $\left(A_{1}, A_{2}\right)$ of rectangles whose height surpasses the unit height over $1 / 8$, they must be in group 4 such that $A_{1}: w=1 / 4, H_{1}=h_{1}+h_{1}^{o}, h_{1}^{o}>1 / 8 ; A_{2}: w=$ $1 / 4, H_{2}=h_{1}+h_{2}^{o}, h_{2}^{o} \leq h_{1}^{o}$; and $1 / 4 \leq H_{2} \leq H_{1}<4 / 9, h_{1} \geq h_{2} \geq 0.5\left(h_{1}^{o}+h_{2}^{o}\right)>1 / 8$ (See Figure 2).

Placement: Place $A_{1}$ on the right and $A_{2}$ on the left.

Shaping: Consider $A_{1}$ first

(1) If $h_{1}+h_{1}^{o}>2 h_{2}$, the area of rectangle $A_{1}$ is bigger than the area of the empty part in the right, set: $\overline{w_{1}}=\frac{3}{4} \sqrt{H_{1}}<\frac{3}{4} \sqrt{\frac{4}{9}}=\frac{1}{2}, \overline{H_{1}}=\frac{4}{9} \overline{w_{1}}=\frac{1}{3} \sqrt{H_{1}}<\frac{1}{3} \sqrt{\frac{4}{9}}=\frac{2}{9}$ (We set the width of as big as possible, so lower the height). The remaining rectangles are packed on the left, so we do not need to make the width of empty part on the right that is integer times of $1 / 8$.

Remark 2: In this case, the shadow area in Fig. 2 does not need to be used to place the remaining rectangles.

Accounting: As $h_{2}>1 / 8$, so we have: $\overline{H_{1}}-h_{2}<2 / 9-1 / 8<1 / 8$.

(2) If $h_{1}+h_{1}^{o} \leq 2 h_{2}$, set $\overline{w_{1}}=3 / 8, \overline{H_{1}}=(2 / 3) \cdot H_{1}$.

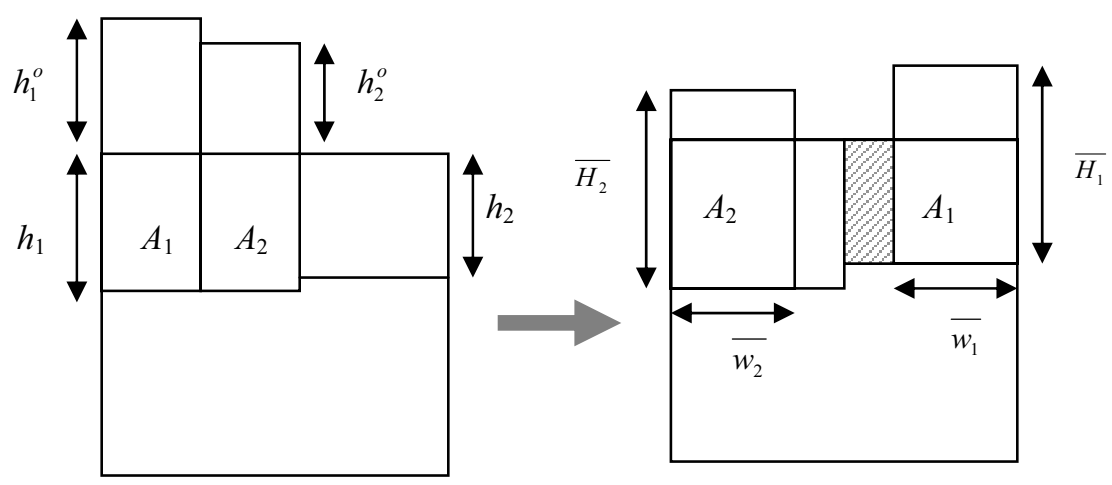

Fig. 2. Shaping in case 1.1 
Remark 3: In this case, the shadow area can be used to pack the remained rectangles. The idea in the Remark 2 and 3 is used in the later cases.

Accounting: $\frac{\overline{H_{1}}}{\overline{w_{1}}}=\frac{16}{9} H_{1} \geq \frac{16}{9} \cdot \frac{1}{4}=\frac{4}{9}$, and $\overline{H_{1}}-h_{2} \leq \frac{2}{3} H_{1}-\frac{1}{2} H_{1}=\frac{1}{6} H_{1}<\frac{2}{27}<\frac{1}{8}$. $A_{2}$ is shaped with $\overline{w_{2}}=\frac{3}{8}, \overline{H_{2}}=\frac{2}{3} H_{2}$.

Accounting: $\frac{\overline{H_{2}}}{\overline{w_{2}}}=\frac{16}{9} H_{2} \geq \frac{16}{9} \cdot \frac{1}{4}=\frac{4}{9}$, and $\overline{H_{2}}-h_{1} \leq \frac{2}{3} H_{2}-\frac{1}{2} H_{2}=\frac{1}{6} H_{2}<\frac{2}{27}<\frac{1}{8}$.

\subsection{Packing Group 6 and Groups $n(n \geq 7)$}

Group $6: w=1 / 8,1 / 8 \leq H<1 / 4$. We first pack group 6 without shaping. The columns with the same bottom can be exchanged freely. Since $(1 / 2) \cdot H<1 / 8$, there are at most three columns whose tops surpass the unit height over $1 / 8$ (totally eight columns). If there are three columns whose tops surpass the unit height over $1 / 8$, then there are at least four columns whose tops below the unit height. First, remove the three rectangles which make the tops of the three columns surpass the unit height over $1 / 8$. Then there are seven columns whose tops below the unit height. Therefore, there always exist three pairs of adjacent columns whose tops are below the unit height. Second, place these three rectangles on the three pairs of columns, and shape them as:

$\bar{w}=\left\{\begin{array}{ll}\frac{3}{2} \sqrt{A}, & \frac{1}{64} \leq A \leq \frac{1}{36} \\ \frac{1}{4}, & \frac{1}{36} \leq A \leq \frac{1}{32}\end{array}, \bar{H}=\frac{A}{\bar{w}} \leq \frac{1}{8}\right.$ (Only in cases 1.2.1(b) and 1.4.1(a), we need additional height $1 / 8+0.006=13.1 \%)$.

Consider the groups $n(n \geq 7)$ with height less than $1 / 8$. For each packing step, we leave enough space and proper width to pack the remaining rectangles. Therefore, packing the groups $n(n \geq 7)$ without any shaping can not make the tops surpass the unit height over $12.5 \%$ or $13.1 \%$.

From the above discussion, we can draw the conclusion that, given a set of soft rectangles of total area $A_{\text {total }}$, maximum area $A_{\max }$, and shape flexibility $r \geq 2.25$, there exists a slicing floorplan $\mathrm{F}$ of these rectangles such that $\operatorname{Area}(F) \leq 1.131 A_{\text {total }}$.

\subsection{An Upper Bounds with Large Flexibility $r$ or Small Maximum Area $A_{\max }$}

In the above analysis, we do not take into account the relative sizes of the rectangles. It should be reasonable to predict a better packing if all the rectangles are small comparing with $A_{\text {total }}$. We consider the following strategy.

The floorplan $F$ is initially divided into columns of width $W=\sqrt{\frac{r A_{\max }}{2}}$. We classify the areas into groups such that area $A$ is in group $i$ when $\frac{W^{2}}{4^{i-1} r} \leq A<\frac{W^{2}}{4^{i-2} r}$ for $i=$ $1,2,3, \ldots$ (See Table 2). An area $A$ from group $\mathrm{i}$ is represented as a rectangle $R$ of width $\frac{W}{2^{i-1}}$ and height $\frac{2^{i-1} A}{W}$. We first pack group 1 , then group 2, and so on, finally group $n(n>2)$ without any shaping. We pack the rectangles one at a time from the largest to the smallest and on the lowest possible level. The width of floorplan $F$ is given by $X=\left[\frac{\sqrt{A_{\text {total }}}}{W} \times W\right.$, which makes the width less than or equal to the height and makes the ratio of the height to the width small. We set $H=A_{\text {total }} / X$. 
Table 2. Classification of Areas in Theorem 3

\begin{tabular}{cccc}
\hline Group & Area $A$ & Width $w$ & Height $H$ \\
\hline 1 & $W^{2} / r \leq A \leq A_{\max }$ & $w=W$ & $W / r \leq H \leq 2 W / r$ \\
\hline 2 & $W^{2} / 4 r \leq A<W^{2} / r$ & $w=W / 2$ & $W / r \leq H<2 W / r$ \\
\hline 3 & $W^{2} / 16 r \leq A<W^{2} / 4 r$ & $w=W / 4$ & $W / 2 r \leq H<W / r$ \\
\hline 4 & $W^{2} / 64 r \leq A<W^{2} / 16 r$ & $w=W / 8$ & $W / 4 r \leq H<W / 2 r$ \\
\hline$\ldots$ & $\ldots$ & $\ldots$ & $\ldots$ \\
\hline
\end{tabular}

We first pack the group 1: $w=W, W / r \leq H \leq 2 W / r$. We use a similar packing technique as case 3.3. We first pack group 1 without shaping. The columns can be exchanged freely. The number of the columns whose tops surpass the height $H$ over $W /$ $r$ is smaller than the total number of the columns whose tops are below the height $H$. By exchanging the columns, we can make the top of the adjacent column of the column whose top surpasses the height $H$ over $W / r$ below the height $H$. Shaping the rectangle as: $\bar{w}=\sqrt{r A}<2 W, \bar{H}=\bar{w} / r=\sqrt{A / r}<W / r$. The remaining rectangles are packed on the other columns.

We now pack the group 2. The Lemma 1 holds with the flexibility $r \geq 3 \sqrt{2} / 2=$ 2.1213. In terms of Lemma 1, we can always compress a pair of modules of group 2 every time, aligned with the same height and the same total width. If the height of the pair is over the height $H$ by $W / r$, we pack the two modules on two columns respectively, and the shaping as we use for group 1.

Finally, we pack the remaining modules without any shaping. The height of the remaining is less than $W / r$. Through the above packing, we have:

$$
\frac{\operatorname{Area}(F)}{A_{\text {total }}} \leq \frac{H+W / r}{H}=1+\frac{W}{r H} \leq 1+\beta, \quad \text { where } \quad \beta=\sqrt{\frac{A_{\max }}{2 r A_{\text {total }}}} .
$$

And we have

$$
1 \leq \frac{\operatorname{height}(F)}{\operatorname{width}(F)} \leq \frac{H+W / r}{X} \leq(1+\beta) \frac{A_{\text {total }}}{X^{2}},
$$

where

$$
W=\sqrt{\frac{r A_{\max }}{2}}, X=\left[\frac{\sqrt{A_{\text {total }}}}{W}\right] \times W\left(\leq \sqrt{A_{\text {total }}}\right), \quad \text { and } \quad H=\frac{A_{\text {total }}}{X} .
$$

\section{Conclusion}

We presented a theoretical upper bound for slicing floorplans with soft rectangles. We proved that, given a set of soft rectangles of total area $A_{\text {total }}$, maximum area $A_{\max }$, and shape flexibility $r \geq 2.25$, there exists a slicing floorplan $\mathrm{F}$ of these rectangles such that $\operatorname{Area}(F) \leq \min \{1.131,(1+\beta)\} A_{\text {total }}$, where $\beta=\sqrt{\frac{A_{\max }}{2 r A_{\text {total }}}}$. 


\section{References}

1. F.Y. Young and D.F. Wong: How good are slicing floorplans. Integration, the VLSI Journal, vol. 23 (1997) 61-73.

2. D.F. Wong and C. L. Liu: A new algorithm for floorplan designs. Proceedings of the $23^{\text {rd }}$ ACM/IEEE Design Automation Conference (1986) 101-107.

3. R.H. J.M. Otten: Automatic floorplan design. Proceedings of the 19th ACM/IEEE Design Automation Conference (1982) 261-267.

4. G.K.H. Yeap and M. Sarrafzadeh: Sliceable floorplaning by graph dualizationn. Siam J. Discrete Math., 8(2) (1995) 258-280.

5. L. Stockmeyer: Optimal orientation of cells in slicing floorplan designs. Information and Control, 57 (1983) 91-101.

6. M. Sarrafzadeh: Transforming an arbitrary floorplan into a sliceable one. Proceedings of the International Conference on Computer-Aided Design (1993) 386-389.

7. N. Sherwani: Algorithms for VLSI physical design automation. Kluwer Publishers (1995).

8. Helvio P. Peixoto, Margarida F. Jacome and Ander Royo: A tight area upper bound for slicing floorplans. Proceedings of $13^{\text {th }}$ International conference on VLSI Design (2000) 280-285.

9. F.Y. Young, D.F. Wong and Hannah H. Yang: Slicing Floorplans with boundary constraints. IEEE Transactions on CADs, vol.18, no.9 (1999) 1385-1389.

10. Weiping Shi: A fast algorithm for area minimization of slicing floorplans. IEEE Transactions on Computer-Aided Design of Integrated Circuits and Systems, 15(12) (1996) 1525-1532.

11. Parthasarthi Dasgupta and Susmita Sur-kolay: Slicing rectangular graphs and their optimal floorplans. ACM Transactions on Design Automation of Electronic Systems, vol.6, no.4, (2001) 447-470.

12. Jin $\mathrm{Xu}$, Pei-Ning Giuo, and Chung-Kuan Cheng: Sequence-pair Approach for rectilinear module placement. IEEE Transactions on Computer-Aided Design of Integrated Circuits and Systems, vol.18, no.4, (1999) 484-493.

13. P. Pan, W. Shi, and C.L. Liu: Area minimization for hierarchical floorplans. Algorithmica, vol.15, no.6, (1996) 550-571.

14. http://www. eetimes.com/story/OEG2 0030110 S 0042 .

15. Guowu Yang etc: A Theoretical Upper Bound for Slicing Floorplan with Soft Modules. Technical report, Portland State University, 2004. www . ece.pdx . edu/ song 